\title{
El agua como servicio de alojamientos turísticos: análisis léxico de páginas web comerciales en lengua francesa ${ }^{1}$
}

\section{Baynat Monreal, $\mathbf{M}^{\mathrm{a}}$ Elena}

ILUMA - Universidad de Valencia. melena.baynat@uv.es

\begin{abstract}
Resumen
El agua es un bien preciado en la promoción de alojamientos turísticos. Todo alojamiento de una categoría media o superior ofrece, al menos, habitaciones con cuarto de baño. $Y$, a medida que va aumentando la categoría del establecimiento, prolifera la sofisticación de sus accesorios. Otra instalación hotelera frecuente que depende totalmente del agua es la piscina: su presencia y descripción suele ser uno de los leitmotiv publicitarios. Hallamos una gran tipología. En último lugar, están los servicios basados en el agua ofrecidos por hoteles con zona spa (cada vez más frecuentes) o los llamados hoteles spa que ofrecen tratamientos relacionados con el agua: estos se convierten en un buen reclamo para los clientes. Para llevar a cabo esta investigación nos basaremos en un corpus de aproximadamente un millón de palabras en lengua francesa compilado de páginas web comerciales de promoción de alojamientos de Francia y Canadá. Analizaremos el léxico relacionado con las instalaciones y servicios basados en el uso del agua citados. Con el estudio cuantitativo y cualitativo de este campo léxico en nuestro corpus -palabras simples y compuestas- esperamos llegar a conclusiones pertinentes sobre el protagonismo del elemento líquido en la promoción hotelera y el papel fundamental de la riqueza léxica del lenguaje publicitario en la captación de clientela.
\end{abstract}

Palabras clave: Turismo e Internet; promoción turística; Lingüística de corpus; Lexicología.

\section{Résumé}

L'eau est une richesse prisée dans la promotion d'hébergements touristiques. En effet, quelques installations et services fréquents en dépendent: tout hébergement d'une catégorie moyenne ou supérieure offre, au moins, des chambres avec une salle de bains. La sophistication de ces installations se multiplie au fur et à mesure que la catégorie de l'établissement augmente. Une autre installation hôtelière fréquente qui dépend totalement de l'eau est la piscine: sa présence et description est souvent un des leitmotiv de la promotion des hébergements. Nous trouvons des piscines de toute sorte. En dernier lieu, nous citerons les services basés sur l'eau, principalement des hôtels avec zone spa (de plus en plus fréquents) ou encore ceux qu'on nomme hôtels spa. L'offre de traitements en rapport avec l'eau y est de plus en plus complète et devient fondamentale pour l'attrait des clients. Pour mener à bout cette recherche nous nous baserons sur un corpus d'environ un million de mots en langue française provenant de sites web commerciaux de promotion d'hébergements en France et au Canada. Nous analyserons le lexique du corpus par rapport aux installations et services basés sur l'utilisation de l'eau cités auparavant. Après avoir réalisé une analyse quantitative et qualitative de ce champ lexique dans notre corpus -mots simples et composés- nous espérons arriver à des conclusions pertinentes sur le rôle principal de cet essentiel élément liquide dans la promotion hôtelière et l'influence fondamentale de la richesse du lexique du langage publicitaire pour attirer de la clientèle.

Mots-clés: Tourisme et Internet; promotion touristique; Linguistique de corpus; Lexicologie.

\footnotetext{
${ }^{1}$ Investigación realizada con la ayuda del Proyecto competitivo COMETVAL: Análisis léxico y discursivo de corpus paralelos y comparables (español, inglés y francés) de páginas electrónicas de promoción turística. FFI2011-24712, concedido por el Ministerio de Economía y Competitividad, en 2012-2014.
} 


\begin{abstract}
Water is a valued benefit in promotions for accommodations in the tourism industry. In fact, some of the most utilized facilities and services depend on water: all middle or first class accommodations offer, at least, rooms with bathrooms. The refinement of these facilities will increase with more prestigious lodgings. Another frequent hotel facility that depends on water is the swimming-pool: its presence and description are some leitmotiv of accommodations' promotion. We find all kinds of swimming-pools. Finally, this study examines the services based on water, mainly offered by hotels with spas namely Spa Hotels. In most of them, water treatment services are increasingly being offered and becoming essential for attracting clients. This research study will be based on a corpus of approximately one million words in French taken from commercial web pages for accommodations promotions in France and Canada. We'll analyse the corpus lexical related to facilities and services based on water use that we mentioned before. After analysing quantity and quality of this lexical field in our corpus -simple and compound words- we hope to obtain pertinent conclusions about the importance of this essential liquid element in hotel promotions and its basic rule in the lexical wealth of advertisement language for attracting clients.
\end{abstract}

Keywords: Tourism and Internet; Tourism promotion; Corpus linguistics; Lexicology.

\title{
Introducción
}

Actualmente no se concibe la promoción turística sin Internet. Han proliferado nuevos géneros discursivos o cibergéneros que, en realidad, no hacen más que reproducir, en su mayoría, los géneros ya existentes en los medios de promoción tradicionales (Crowston y Willians, 2000: 201-215) con la particularidad añadida de que al ser digitales ofrecen textos actualizables, más accesibles y flexibles, promoviendo la comunicación bilateral.

Según Sanmartín «la red se ha convertido en una especie de agencia de viajes, en un sistema de promoción publicitaria de lugares y emplazamientos, en un punto de encuentro para conversar sobre las experiencias del viaje y, por supuesto, en un marco de interacción comunicativa inédito y apasionante para el estudioso del lenguaje» (2010:10). En efecto, Internet es un espacio privilegiado donde promocionar productos turísticos, informarse o reservarlos.

En este artículo nos centraremos en el estudio de un género fundamental del sector: las páginas web privadas de promoción turística, clasificadas como géneros comerciales «que se desarrollan en los departamentos de marketing de las agencias y otras empresas de turismo, destinados a promover la venta directa del producto turístico, conquistando la mente del consumidor» (Calvi, 2010: 22). Más concretamente, analizaremos sitios web de promoción de alojamientos en lengua francesa. Para ello nos basaremos en un corpus comparable almacenado en la base de datos trilingüe COMETVAL creada por el grupo de investigación del mismo nombre²

El uso de un corpus extraído de la web se justifica por la necesidad de obtener «muestras de habla empleadas en su contexto de uso» obtenidos de «textos representativos y reales» (Sanmartín, 2012: 128) que nos permitan analizar el auténtico discurso turístico.

Observaremos el comportamiento sintáctico y semántico de las unidades léxicas (simples y compuestas) del corpus relacionadas de un modo u otro con el agua en su contexto de uso. Analizaremos las combinaciones léxicas más frecuentes.

Tras realizar la clasificación de las unidades y combinaciones léxicas y fraseológicas empleadas en contextos reales, estudiaremos de qué modo puede su uso imaginativo ser decisivo para una promoción de productos turísticos más original, directa y eficaz. Con este modesto estudio lexicográfico esperamos ayudar a mejorar la creación y traducción de páginas web de promoción de alojamientos.

\footnotetext{
${ }^{2}$ Frutos del trabajo del grupo de investigación COMETVAL (IULMA-Universidad de Valencia) son el Diccionario Multilingüe de Turismo (2014) y la Guía de buenas prácticas discursivas (2016) que propone estrategias lingüísticas para la promoción turística en Internet (cf. Referencias bibliográficas.)
} 


\section{Lexicología, fraseología, compuestos y colocaciones}

La lexicografía es relativamente reciente como disciplina independiente. Sin embargo, el aspecto que más ha llamado la atención de lingüistas e investigadores de diferentes disciplinas no es el estudio del léxico aislado sino «la restricción combinatoria, ya sea léxica o sintáctica» (Corpas Pastor, 2001: 89), es decir, el ámbito de la fraseología.

Corpas Pastor considera que la fraseología es una sub-disciplina de la lexicología, sin embargo, existe controversia sobre su ubicación. Otros autores como Skolnikova (2010: 7) o González (2006: 2) afirman que podría ser una rama de estudio independiente ocupando, incluso, el mismo nivel que la lexicología. En cualquier caso, ambas están interrelacionadas y se necesitan puesto que se preocupan del mismo objeto de estudio (el léxico) con la diferencia que la primera es más específica: se centra en el estudio concreto de «las combinaciones de palabras o unidades fraseológicas» (Corpas Pastor, 1996: 170).

En esta investigación nos interesan las dos, independientemente de su ubicación, puesto que estudiaremos el léxico del agua, su frecuencia y su comportamiento semántico en nuestro corpus pero también las unidades fraseológicas relacionadas con este elemento o que lo incluyan, principalmente los compuestos y las colocaciones en los que aparezca como componente. Diferenciaremos, en términos generales, entre compuestos y colocaciones: los primeros funcionan como unidades lexicalizadas independientes mientras que los segundos pueden ser considerados como frases, es decir, que existe una relación sintáctica entres sus unidades. Además, un compuesto tiene un significado unitario mientras que en una colocación o semi-frasema el significado puede deducirse de la suma de sus componentes y el colocado o colocativo (B) depende de su base (A), la cual tiene autonomía semántica y determina la elección del colocado. Este último, es, a su vez, intercambiable por otros colocados que añaden diferentes matices semánticos a la base, de carácter principalmente abstracto o figurativo (Skolnikova, 2010: 17-22).

Resumiendo, analizaremos las unidades léxicas simples y compuestas seleccionadas observando su frecuencia, su comportamiento morfo-sintáctico y su significado. Nos centraremos en los compuestos y colocaciones relacionados con el medio acuático, analizando sus componentes así como sus relaciones contextuales.

\section{Presentación del corpus}

Nuestro corpus está formado por aproximadamente un millón de palabras compilado a partir de páginas web de alojamientos en lengua francesa de Francia y Canadá. Es un corpus más o menos equilibrado y variado en cuanto a número de alojamientos de ambos países, tipos (hoteles, campings o alojamientos rurales), categoría, distribución geográfica (diferentes zonas) o tipología (generales, temáticos o dirigidos a diferentes tipos de públicos). Sin embargo, hemos seleccionado también, en número similar a otra categoría de hoteles, los campings y alojamientos rurales; en consecuencia, en el corpus hay una mayor cantidad de hoteles que de otro tipo de alojamientos, hecho que puede condicionar o variar los resultados de nuestro análisis ${ }^{3}$.

Para seleccionar el léxico que nos interesa (en relación con el agua) utilizaremos la herramienta antonc ${ }^{4}$ que nos permite obtener números de frecuencias de unidades simples y compuestas o detectar sus principales concordancias y colocaciones.

Analizaremos la unidad léxica simple eau y sus colocaciones pero también todo el vocabulario del corpus relacionado con ella que hemos subdividido en tres bloques, relacionados con tres instalaciones frecuentes en los alojamientos que necesitan el agua u ofrecen servicios relacionados con ella:

a. Las instalaciones sanitarias de las habitaciones.

b. La piscina.

c. El spa

También existen otras instalaciones o servicios del hotel no recogidos en estos tres bloques que emplean el agua, menos frecuentes en el corpus; por ejemplo: el servicio de tintorería, el agua embotellada como producto de cortesía o el servicio de vaciado de aguas negras para caravanas en los campings.

\footnotetext{
${ }^{3}$ Somos conscientes de partir de un corpus limitado e incompleto que, aunque por su tamaño es representativo de la tendencia general de las páginas web de alojamientos, no es científicamente comprobable en el sentido de que no estamos analizando todos los tipos de alojamientos ofertados en la web sino solamente una muestra de ellos.

${ }^{4}$ Programa de frecuencias y concordancias disponible en la web.
} 
A continuación clasificaremos el léxico acuático más frecuente del corpus centrándonos principalmente en los tres bloques citados, aunque compartirán algunos elementos. Por ejemplo, existen duchas en los cuartos de baño de las habitaciones pero también en la piscina o encontramos bañeras de hidromasaje en el spa, pero también en las habitaciones o incluso en algunas piscinas.

\section{El agua}

El agua es un elemento básico de cualquier alojamiento, necesario para ofrecer a la clientela servicios de aseo y relax. Entre las unidades léxicas simples más frecuentes del corpus observamos que la palabra eau ocupa el puesto 77 y tiene una frecuencia alta (1.453 fr.). También hallamos otras unidades simples frecuentes relacionadas con el mismo campo semántico; entre los sustantivos comunes extraído con el programa antconc obtenemos una selección muy representativa de términos clave para la promoción hotelera:

Tabla 1. Sustantivos comunes más frecuentes del corpus

\begin{tabular}{|c|c|}
\hline Unidad Simple & $\mathbf{N}^{0}$ de Frecuencias \\
\hline 1. $\quad$ chambre/chambres $^{5}$ & $4528+4079=8.607$ \\
\hline 2. hôtel/hotel/hôtels/hotels ${ }^{6}$ & $5.320+1.702+380+145=7.537$ \\
\hline 3. personne/pers/personnes ${ }^{7}$ & $1.265+1.761+2.381=5.407$ \\
\hline 4. salle/salles & $3.731+940=4.671$ \\
\hline 5. service/services & $2.176+2.320=4.496$ \\
\hline 6. Lit/lits & $3.423+1.041=4.464$ \\
\hline 7. accès & 2.753 \\
\hline 8. bain/bains & $2.204+432=2.636$ \\
\hline 9. séjour/séjours & $2.313+265=2.578$ \\
\hline 10. Internet & 2.239 \\
\hline 11. suite/suites & $1.331+972=2.303$ \\
\hline 12. forfait/forfaits & $1.573+647=2.220$ \\
\hline 13. activité/activités & $290+1.899=2.189$ \\
\hline 14. nuit/nuits & $1.313+766=2.079$ \\
\hline 15. déjeuner/déjeuners & $2.022+343=2.055$ \\
\hline 16. centre/centres & $1.932+99=2.031$ \\
\hline 17. vue/vues & $1.442+84=1.526$ \\
\hline 18. kilomètre/kilomètres/kmt & $32+105+1.375=1.512$ \\
\hline 19. eau/eaux & $1.363+90=1.453$ \\
\hline 20. ville/villes & $1.362+57=1.419$ \\
\hline
\end{tabular}

Fuente: creación propia

\footnotetext{
${ }^{5}$ Hemos lematizado masculinos y femeninos para que nuestro análisis muestre resultados más reales de uso.

${ }^{6}$ Se ha encontrado la palabra hotel mal escrita (sin el acento circunflejo) en 1702 ocasiones en el corpus. Por su elevada frecuencia las contabilizamos también, considerando que han sido simples errores tipográficos de descuido de los creadores de las páginas.

${ }^{7}$ Hemos contabilizado también algunas abreviaturas frecuentes en el corpus como per.s o kmt.
} 
En el cuadro 1, tras varios sustantivos relacionados con las instalaciones y el mobiliario del alojamiento, la clientela y los servicios, en octavo lugar aparece una unidad simple del campo semántico del agua: bain (más frecuente que eau). Bain pertenece al primer bloque temático de nuestra clasificación: las instalaciones sanitarias, elemento esencial de cualquier alojamiento. Su elevada frecuencia en el corpus nos lleva a presuponer que este primer bloque es el más relevante de nuestro estudio.

Después de bain, hallamos algunos términos de otro campo semántico fundamental en los alojamientos: la gestión (séjour y forfait) seguidos de Internet (2.239 fr.), sustantivo esencial por ser el principal medio elegido en la actualidad para la promoción y gestión de los servicios hoteleros.

Finalmente, el sustantivo eau (1.453 frecuencias) ocupa el puesto 19. Veremos a continuación en qué contextos de uso y con qué otras unidades aparece en nuestro corpus.

Tabla 2. Compuestos de eau más frecuentes del corpus

\begin{tabular}{lll}
\multicolumn{1}{c}{ Unidad Compuesta } & No de Frecuencias \\
\hline salle/s d'eau & 647 & \\
eau chaude & 48 \\
plan/s d'eau & 18 \\
bouteille/s d'eau & 10 \\
point d'eau & 7 \\
consommation d'eau & 6 \\
eau chauffée & 4 \\
glissades d'eau & 3 \\
jardins d'eau & 3 \\
jeux d'eau & \\
\hline
\end{tabular}

Fuente: creación propia

En la mayoría de compuestos de la palabra eau más frecuentes hallados en el corpus (cuadro $\mathrm{n}^{\circ} 2$ ) encontramos la misma composición gramatical (sustantivo+preposición+sustantivo) en la que la palabra agua es siempre el segundo sustantivo (B), es decir el que complementa al primero (A): salle d'eau, plan d'eau, bouteille d'eau, point d'eau, consommation d'eau, glissades d'eau, jardins d'eau y jeux d'eau. Solamente se usa otra estructura en dos ocasiones (sustantivo+adjetivo): en este caso el nombre que nos ocupa adquiere más relevancia convirtiéndose en el primer elemento de la unidad (A) acompañado por un adjetivo calificativo (B) que hace función de determinante: eau chaude y eau chauffée.

Como se observa en el cuadro precedente hay varios compuestos del primer tipo citado (sust.+prep.+sust.) en los que el primer elemento (A) puede aparecer en singular o en plural indistintamente, sin embargo en consommation d'eau solo se emplea en singular y, al contrario, en glissades d'eau o jardins d'eau únicamente en plural.

Sin embargo, el plural de eau se repite únicamente en 90 ocasiones en el corpus: 
Tabla 3. Compuestos de eaux más frecuentes del corpus

\begin{abstract}
eaux ménagères (12 frecuencias) y eaux vannes (4 frecuencias) ${ }^{8}$ : servicio de vaciado de aguas limpias y sucias para caravanas o autocaravanas en campings.

eaux minérales (6 frecuencias): servicio de agua embotellada en alojamientos.

eaux vives (7 frecuencias): espacio, normalmente exterior al alojamiento, para realización de actividades de aventura.
\end{abstract}

Fuente: creación propia

Todas las unidades recogidas en el cuadro 3 ofrecen la misma composición gramatical: sustantivo+ adjetivo.

Volviendo al cuadro 2, en cuanto a su significado, los compuestos de eau citados se refieren principalmente a las instalaciones y servicios del alojamiento. Por ejemplo salle d'eau y eau chaude (los compuestos más frecuentes) se refieren, normalmente, al cuarto de baño de las habitaciones: primer bloque temático (las instalaciones sanitarias). Sin embargo plan d'eau y point d'eau son elementos que se sitúan en las instalaciones comunes del alojamiento, como pueda ser el hall o el jardín, aunque, en ocasiones, en las páginas web se habla también de los alrededores donde puede haber también pozos o estanques. Por ejemplo: «À deux pas du plan d'eau, terrasse berçé par le bruit des cascades [...]» (Gîte le Noble Quêteux, Baie Saint Paul, Francia). En cualquier caso, lo que se quiere publicitar al destacar este tipo de elementos, tanto en el interior como en el exterior del establecimiento, es una imagen de frescura, relax y bienestar.

Destacaremos, a continuación, cuatro colocaciones frecuentes de salle d'eau: salle d'eau wc/salle d'eau+wc (350 fr.), salle d'eau avec douche (10 fr.), salle d'eau avec lavabo (3 fr.), salle d'eau privative (12 fr.). Retomando nuestro marco teórico, consideramos que salle d'eau es un compuesto y no una colocación porque es una unidad lexicalizada fijada en la lengua por su uso habitual y actúa como un sustantivo. Sin embargo, los últimos cuatro ejemplos citados son colocaciones de esta unidad compuesta puesto que funcionan como frases o sintagmas, es decir que existe una relación sintáctica entre sus unidades; por ejemplo: salle d'eau avec douche (nombre+complemento del nombre) o salle d'eau privative (nombre+ determinante). Además su significado puede deducirse de la suma de sus componentes: se trata de un cuarto de baño (base) al que se le añaden matices semánticos con sus diferentes colocados (con ducha, con lavabo, privado, etc.)

Terminaremos citando tres unidades compuestas de eau poco frecuentes (una frecuencia) pero que consideramos relevantes en la promoción de alojamientos por diferentes motivos. La primera es matelas à eau, la destacamos porque este tipo de accesorios de confort y lujo ofertados por ciertos hoteles de categoría superior para sus habitaciones pueden marcar la diferencia para la captación de un mayor número de clientela exclusiva. En segundo lugar, économiseurs d'eau: últimamente se ha puesto muy de moda que los establecimientos hoteleros sean respetuosos con el medioambiente y tomen medidas para evitar derroches, como este aparato que reduce el gasto de agua: este tipo de acciones suelen usarse como reclamo de clientes en la publicidad de sus alojamientos. Y, por último, branchement eau, un servicio fundamental de los campings dirigido principalmente a los campistas, muy frecuentes en países francófonos, que necesitan agua limpia durante su estancia para llenar los depósitos de la caravana o autocaravana.

\title{
4. Las instalaciones sanitarias
}

Como hemos podido observar, el agua es un elemento esencial de los cuartos de baño de los alojamientos. En el corpus encontramos tres denominaciones sinónimas para estos espacios: bain (2.204 fr.), salle/s de bain/s (1.475 fr.), salle/s d'eau (547 fr.). Su alta frecuencia es signo de su relevancia en las páginas web promocionales. Sin embargo, toilettes se repite únicamente en 61 ocasiones. Debemos destacar el hecho de la gran utilización de bain, muestra de la tendencia de economizar en la lengua favorecida por el uso de internet. En nuestro corpus bain suelto se emplea más en hoteles canadienses que franceses, mientras que salle de bains se utiliza de modo equilibrado entre los dos países y salle d'eau mayoritariamente en hoteles franceses.

${ }^{8}$ También hemos encontrado eaux noires pero con una sola frecuencia en el corpus. 
En cuanto a las instalaciones y accesorios relacionados con el agua incluidos en los cuartos de baño de los alojamientos analizados destacaremos que cuanta más categoría mayor es su especialización y sofisticación. Dos elementos primordiales que no suelen faltar son la douche (1.280 frecuencias) o la baignoire (545 frecuencias), más prescindible que la ducha y, en consecuencia, menos empleada en los ejemplos de nuestro corpus.

Tabla 4. Compuestos de douche más frecuentes del corpus

\begin{tabular}{lll}
\hline \multicolumn{1}{c}{ Unidad Compuesta } & No de Frecuencias \\
\hline douche vitrée & 13 \\
douche multi-jets & 10 \\
cabine de douche & 9 \\
douche hammam & 5 \\
rideau de douche & 5 \\
salle de douche & 2 \\
douche-pluie & 2 \\
douche (ou baignoire) à jets hydro & 2 \\
pare-douche & \\
\hline
\end{tabular}

Fuente: creación propia

Como se observa en el cuadro precedente, la estructura gramatical de las unidades compuestas de douche es más variada que la de eau: sustantivo+adjetivo (douche vitrée), sustantivo+sutantivo (douche hammam, douche-pluie, douche multi-jets) sustantivo+preposición+sustantivo (cabine de douche, rideau de douche, salle de douche), sustantivo+preposición+sustantivo+adjetivo (douche à jets hydro) verbe+sustantif (pare-douche). En cuanto al semantismo se refieren tanto a la instalación (salle de douche), el equiplamiento de esta (cabine de douche) como algunos accesorios fundamentales para que el agua no salpique (rideau de douche o pare-douche) u otros más específicos que favorecen que el momento de la ducha sea más relajante (douche multi-jets, douche-pluie, douche à jets hydro, douche hamam).

Destacaremos otras colocaciones de douche menos frecuentes pero importantes para la clientela con movilidad reducida: douche adaptée (2 fr.), douche (équipée) avec/de barres de soutien (5 frecuencias) o douche avec barre d'appui (1 fr.).

En cuanto a baignoire, es significativamente menos frecuente en el corpus que douche. Esto es debido a que se considera como un equipamiento menos necesario: el baño se suele asociar como un lujo, una actividad más relajante. Prueba de ello es la elevada presencia en el corpus de la unidad lexicológica compuesta baignoire (double) à remous (142 frecuencias) así como la inclusión en algunos cuartos de baño de las habitaciones de otro tipo de bañeras de relax: baignoire balnéo (19 frecuencias), baignoire thérapaeutique (11 frecuencias), baignoire jacuzzi (1 frecuencia).

En cuanto a las colocaciones más frecuentes destacaremos dos con similar composición gramatical: salle de bain avec baignoire (26 frecuencias), bain avec douche et baignoire (12 frecuencias).

Y, para finalizar, hemos seleccionado un compuesto formado por ambos elementos: baignoire-douche (6 frecuencias) que no está recogido como entrada en diccionarios generales de la lengua francesa ${ }^{9} \mathrm{y}$, sin embargo, aparecen cuartos de baño con esta instalación tanto en alojamientos franceses como canadienses:

\footnotetext{
${ }^{9}$ Larousse y Petit Robert, por ejemplo.
} 
Tabla 5. Ejemplo de uso del corpus del compuesto baignoire-douche

\begin{tabular}{ll}
\hline \multicolumn{1}{c}{ Ejemplo } & \multicolumn{1}{c}{ Fuente (página web del hotel) } \\
\hline $\begin{array}{l}\text { La salle de bain est en marbre d’Italie avec baignoire- } \\
\text { douche, lavabo et WC. }\end{array}$ & Hôtel Résidence des Halles, Paris, Francia \\
Salle de bain avec baignoire-douche ou simple douche & Hôtel Campanile Paris Bastille, Paris Francia \\
Salle de bain avec baignoire-douche & Chambre champêtre à l'Auberge du Lac \\
& Taureau, Canadá \\
\hline
\end{tabular}

Fuente: creación propia

\section{La piscina}

Otra instalación común de muchos alojamientos que no puede prescindir del agua es la piscina. Este sustantivo goza también de una elevada frecuencia en nuestro corpus: 1.043 frecuencias. El único compuesto destacable que hemos hallado es bar piscine que aparece solamente en cuatro ocasiones y en el Multilingual Dictionary of Tourism se define del siguiente modo: «Bar situé près de la piscine ou dans certains établissements hôteliers dans la piscine».

Tabla 6. Colocaciones más frecuentes de piscine en el corpus

\begin{tabular}{lll}
\hline \multicolumn{1}{c}{$\begin{array}{c}\mathbf{N}^{\mathbf{0}} \text { de } \\
\text { frecuencias }\end{array}$} & \multicolumn{1}{c}{ Colocaciones } & \multicolumn{1}{c}{ Categorías gramaticales } \\
\hline 203 & piscine intérieure & sust.+adj. \\
163 & piscine extérieure & sust.+adj. \\
40 & Piscine chauffée & sust.+adj. \\
26 & piscine couverte & sust.+adj. \\
8 & piscine olymique & sust.+adj. \\
8 & au bord de la piscine & loc. prep.+art.+sust. \\
4 & en bord de piscine & loc. prep.+sust. \\
2 & en bordure de piscine & loc. prep.+sust. \\
\hline
\end{tabular}

Fuente: creación propia

Como se observa en el cuadro 6 hay dos bloques diferenciados de colocaciones de piscine : el primero (las 5 más frecuentes) que se refieren a tipos de piscinas, tanto por su ubicación como por su temperatura; el segundo (las 3 últimas) formado por expresiones que sirven para destacar que algunos espacios o actividades del hotel está ubicados o son realizados al borde de la piscina. Por ejemplo: «Le Peter’s Bar. En bord de piscine vous dégusterez, l’après midi, glaces [...]» (Le Mas de Pierre, Saint Paul de Vence, Francia) ; «Relaxation au bord de la piscine, douceur et charme provencal [...]» (Hôtel Novotel Marseille Est, Marseille, Francia).

En cuanto a la composición gramatical también observamos dos tipos diferenciados entre los bloques citados.

Sin embargo, debemos matizar que algunas de las colocaciones que hemos contabilizado en el cuadro 6 no tienen todas ellas el mismo emplazamiento. Por ejemplo, alguna de las piscinas climatizadas son de uso general de la clientela: «[...] la piscine chauffée est le lieu idéal pour se délasser après avoir exploré [...]» (Hôtel de la Cité, Carcasonne, Francia); «Nombreuses activités extérieures: piscine chauffée et à l'eau de mer salée (en saison) [...]» (Hôtel la Roche pleureuse, 
Québec, Canada). Pero otras piscinas caldeadas están ubicadas en la zona spa del hotel y, en consecuencia, no suelen ser de entrada libre.

\section{La zona spa}

Para finalizar analizaremos el léxico acuático del corpus empleado en las páginas web promocionales de alojamientos para referirse en la zona de relax y belleza de los hoteles así como a sus servicios, otro espacio donde el preciado líquido es imprescindible.

Como hemos visto, en algunos alojamientos hay piscinas exclusivas de estas zona spa que se suelen denominar con la colocación piscine chauffée (cuadro $\mathrm{n}^{\circ}$ 6), como por ejemplo: «Espace détente avec piscine chauffée de $15 \mathrm{~m}$ de long [...]» (Hôtel Parc Beaumont, Pau, Francia).

Dicha zona, según los ejemplos del corpus, suele llamarse spa (1.008 fr.) o, a veces, espace détente (19 fr.). Las dos colocaciones más frecuentes que hemos encontrado son spa extérieur (52 fr.) y spa nordique (10 fr.), ambas con la misma composición gramatical (nombre+adjetivo).

En este espacio suele haber también otro tipo de instalaciones, todas denominadas con unidades léxicas simples:

La sauna (261 frecuencias) es un espacio que normalmente precisa de reserva previa y pago adicional por parte del cliente para su uso: «L’accès privé au bain tourbillon et sauna (réservation requise - selon disponibilités)» (Auberge des peupliers, La Malbaie, Canadá). Destacaremos una colocación frecuente formada por el sustantivo seguido de un adjetivo: sauna finlandais (15 frecuencias).

Además resaltamos un sustantivo de procedencia árabe, el hammam (91fr.). En las páginas analizadas no se emplea con su sentido original sino para denominar al tratamiento consistente en una variante del spa, es decir un baño de vapor a alta temperatura: «Le hammam est un bain de vapeur à 50 degrés Celsius» (Appalaches Lodge-Spa-Villégiature, SaintPaul de Montminy, Canadá).

El jacuzzi (72 fr.) puede estar incluido en el cuarto de baño privado de la habitación o en la piscina común y no debe faltar en cualquier espacio spa que se precie. Citaremos las siguientes colocaciones: jacuzzi intérieur (4 fr.) y jacuzzi intérieur (1 fr.) que se ubica en las instalaciones comunes del alojamiento así como jacuzzi double (4 frecuencias), situado, normalmente, en la propia habitación: «Suite King. Description. Vu sur la rivière. Balcon ou terrasse avec hamac. Lit king. Foyer. Jacuzzi double» (Auberge et Spa Laurentides Beaux Rêves, Québec, Canada).

Otro sustantivo que denomina un tratamiento de relajación de los espacios de salud de los alojamientos es el watsu ${ }^{10}(14$ frecuencias).

Terminaremos con otros tratamientos acuáticos, diferentes tipos de baños terapéuticos, colocaciones de bain que tienen como colocados un adjetivo (función de determinante) o una preposición y un sustantivo (función de CN): bain tourbillon (143 fr.), bain de vapeur (9 fr.) y bain bouillonnant (8 fr.). Pueden ser disfrutados en la zona spa pero también alrededor de la piscina o incluso en la habitación.

\section{Conclusión}

En el corpus seleccionado se observa un gran número de unidades léxicas y fraseológicas relacionadas con el agua que demuestran la importancia de este elemento para la promoción de este tipo de establecimientos. Las unidades simples bain y eau destacan por su elevada frecuencia, encontrándose entre los primeros 20 sustantivos más empleados en el corpus. Pero, además, hemos podido comprobar que existe un gran número de unidades simples y compuestas frecuentes así como de colocaciones de estas que hemos podido reagrupar en tres bloques temáticos según se refieran a: las instalaciones sanitarias, la piscina o la zona spa. De todos modos debemos destacar que algunas palabras pueden encontrarse en varios de estos bloques, es decir, que los límites no son precisos pero nos han servido para clasificar y analizar mejor las unidades recogidas.

La riqueza de vocabulario y la gran cantidad de unidades fraseológicas halladas refuerzan la tesis de que un alojamiento sin agua no tiene ninguna posibilidad de triunfar ante la competencia y, en consecuencia, toda página web comercial promocional de establecimientos hoteleros contiene vocabulario relacionado con este campo temático: más o menos

\footnotetext{
${ }^{10} \mathrm{El}$ watsu es un masaje terapéutico que se realiza en el agua, el nombre proviene de fusión de -water- con el nombre que recibe un tipo de masaje de origen japonés Shiatsu.
} 
rico y sofisticado, dependiendo de la categoría del alojamiento. Los hoteles más lujosos son los que ofertan más tipos de duchas, bañeras, piscinas, jacuzzis, tratamientos de belleza y relax, etc. Como se suele decir «cuanto más azúcar más dulce». Para ser competitivos los establecimientos deben ofrecer cada vez mejores instalaciones y un mayor número de servicios -cuanto más exclusivos mejor- y además promocionarlos en sus páginas web para captar el máximo de clientela. Y, sin duda, el agua es un elemento primordial incluido o presente en muchos de ellos. En definitiva, el lenguaje publicitario es variado en vocabulario; en los sectores especializados, como el turístico, contiene un elevado número de combinaciones léxicas que lo enriquecen y favorecen su principal objetivo: llamar la atención de los lectores y conseguir que se conviertan en clientes de esos productos ofertados, ganando a la competencia.

\section{Referencias bibliográficas}

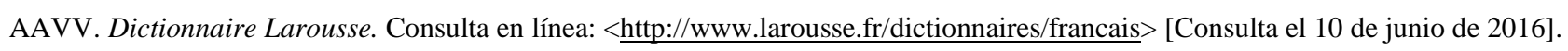

Artusi, Andrea; BAynat, Ma Elena; Dolon, Rosana y otros (2014). Multilingual Dictionary of Tourism Diccionario Multilingüe de Turismo. Consulta en línea: <http://tourismdictio.uv.es/> [Consulta el 10 de junio de 2016].

Artusi, Andrea (coord.); BAynat, Ma Elena; Dolón, Rosana y otros (2016). Guía de buenas prácticas discursivas: estrategias lingüísticas para la promoción turística en la red. Anejo nº de Normas: revista de estudios lingüísticos hispánicos. Consulta en línea: <www.uv.es/normas $>$ [Consulta el 10 de junio de 2016].

CALVI, Ma Vittoria (2010). «Los géneros discursivos en la lengua del turismo:una propuesta de clasificación» en Ibérica 19, 9-32.

CORPAS PASTOR, Gloria (1996): Manual de Fraseología española, Madrid, Gredos.

CORPAS PASTOR, Gloria (2001): «En torno al concepto de colocación» en Euskera, nº 46, 89-108. Consulta en línea:

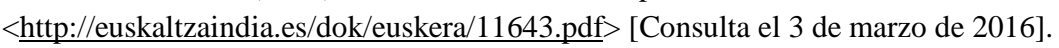

Crowston, Kevin y williams, Marie (2000). «Reproduced and emergent genres of communication on de Word Wide Web», en The Information Society 16, 201-215.

GonZÁLEZ, Fernando (2006). «Las colocaciones en la enseñanza del español de los negocios» en Revista de Didáctica MarcoELE $n^{\circ}$

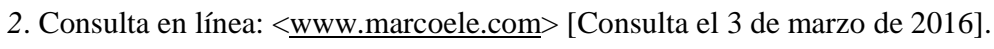

RoBert, Paul (1986). Petit Robert: Dictionnaire de la langue française. Paris: Le Robert.

SANMARTin, Julia (2012). Discurso turístico e internet. Madrid: Ibeoramericana Verbuet

SANMARTín, Julia (2012). «Aplicaciones lexicográficas de un corpus de discurso turístico: contextos de uso y definiciones» en Pasos. Revista de turismo y patrimonio cultural, 128-145.

SкоLnikova, Pavlina (2010). Las colocaciones en el español actual. Consulta en línea: $<$ http://is.muni.cz/th/145385/ff_m/las_colocaciones_lexicas_en_el_espanol_actual.pdf > [Consulta el 3 de marzo de 2016].

\section{Hoteles citados}

Auberge DES Peupliers. Auberge des peupliers. La Malbaie (Canadá). Consulta en línea:

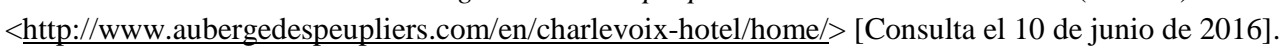

Auberge du LaC TAureau. Chambre champêtre à l'Auberge du Lac. Taureau (Canadá). Consulta en línea:

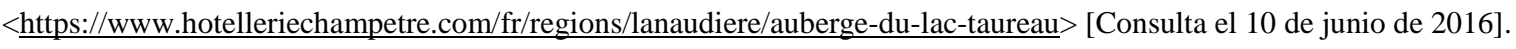

Auberge Spa Beaux Reves. Auberge et Spa Laurentides Beaux Rêves. Québec (Canada). Consulta en línea:

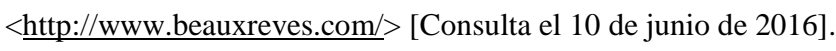

Gite Le Noble Queteux. Gîte le Noble Quêteux. Baie Saint Paul (Francia). Consulta en línea: < http://www.noblequeteux.com/> [Consulta el 10 de junio de 2016].

Hotel Appalaches. Appalaches Lodge-Spa-Villégiature. Saint-Paul de Montminy (Canadá). Consulta en línea:

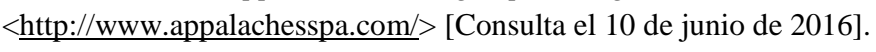

Hotel Campanile. Hôtel Campanile Paris Bastille, Paris Francia. Consulta en línea: <http://www.campanile-parisbastille.fr/eshttp://www.noblequeteux.com/> [Consulta el 10 de junio de 2016].

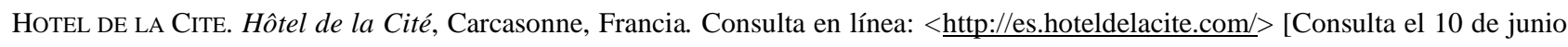
de 2016]. 
Hotel La Roche Pleureuse. Hôtel la Roche pleureuse. Québec (Canada). Consulta en línea: < http://www.rochepleureuse.com/> [Consulta el 10 de junio de 2016].

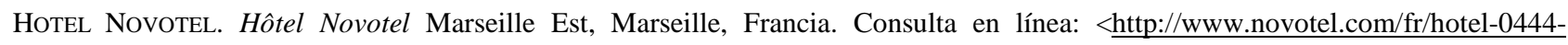
novotel-marseille-est/index.shtml $>$ [Consulta el 10 de junio de 2016].

Hotel Parc Beaumont. Hôtel Parc Beaumont. Pau (Francia). Consulta en línea: <http://www.hotel-parcbeaumont.com/index.html?L=0 [Consulta el 10 de junio de 2016].

Hotel Residence Des Halles. Hôtel Résidence des Halles, Paris, Francia. Consulta en línea: < http://www.hallehotel.fr/> [Consulta el 10 de junio de 2016].

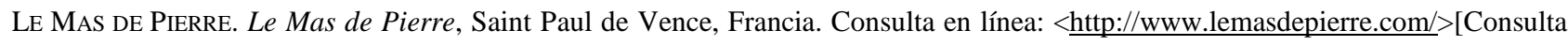
el 10 de junio de 2016]. 